\title{
Quality of Service Aspects of Transport Technologies for the UMTS Radio Access Network
}

\author{
Heba Koraitim, Günter Schäfer, Samir Tohmé \\ Ecole Nationale Supérieure des Télécommunications, Paris, France \\ Email: [Samir.Tohme|Heba.Koraitim|Guenter.Schaefer]@enst.fr
}

Key words: Quality of Service, UMTS, RAN, AAL-2, IP, Satellite Communications

Abstract: The radio access network of upcoming third generation mobile communication systems is currently a subject of intense research and standardization efforts. This article discusses in the first part quality of service aspects of the realization of the transport network using the currently most concurring technologies for this issue: ATM adaptation layer 2 (AAL-2) and IP. The second part of the paper considers architectural alternatives for realizing the radio access network with satellite communications.

\section{INTRODUCTION}

The upcoming $3^{\text {rd }}$ generation mobile communication system UMTS is envisaged to be employed in a wide range of scenarios, as depicted in figure 1. The design of its' radio access network (RAN) is currently subject to intense research and standardization efforts. The first approach for the terrestrial radio access network (UTRAN) currently developed by the $3^{\text {rd }}$ Generation Partnership Project uses ATM as a proven technology for transport in the fixed part of the RAN. An alternative, very recent development is to consider an All-IP architecture combined with a quality of service (QoS) framework for this. The specification of a satellite RAN is quite open up to now. This paper presents the current state concerning transport technologies to be deployed in the fixed part of the terrestrial RAN and considers different architectues for a satellite RAN. Special attention is given to the QoS aspects in the RAN. 


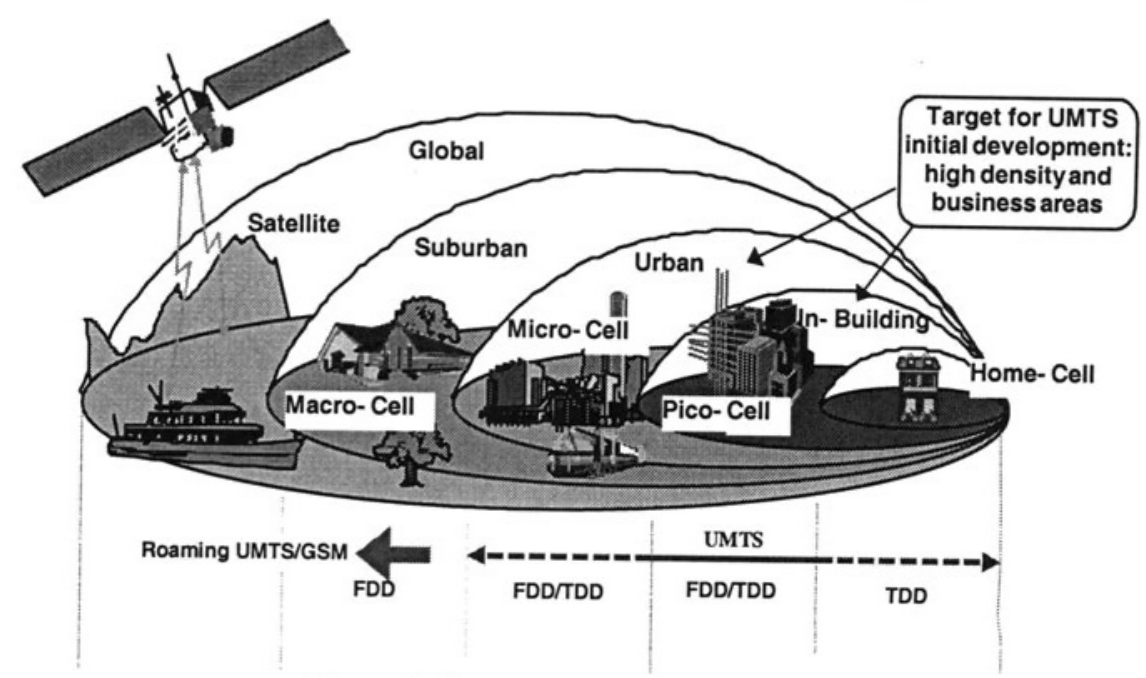

Figure 1: Scenarios for UMTS deployment

\section{QOS IN UMTS AND THE RAN}

The quality of service concept [5] developed in the $3^{\text {rd }}$ Generation Partnership Project is based on the fundamental idea, that the user cares only about issues that are visible to him. As following this idea only the QoS perceived by end-users matters, it has to be provided end-to-end. Furthermore, the number of parameters to be defined or controlled by the user should be as small as possible, the derivation of QoS attributes from the application requirements should be simple and the QoS attributes should be able to support the asymmetric nature of some applications concerning the uplink and downlink direction.

The UMTS standardization is a continuos process and so the specifications are organised as a series of releases. The UMTS release ' 99 assumes the QoS related bearer definitions of GSM to be sufficient and aims to provide the services speech, non-transparent data, and transparent data.

The QoS Architecture proposed by 3GPP to support the QoS requirements of these services distinguishes different levels of QoS according to a layered architecture of bearer services in which services of one layer make use of the services of lower layers.

As user traffic passes from one terminal equipment (TE) to another it passes different bearer services of the network(s). The UMTS QoS architecture leaves aside the bearer services outside the scope of its' standardization and, therefore, concentrates on the UMTS bearer service and the bearer services this service is build upon. 
Taking into account the restrictions and limitations of the air interface, which due to different error characteristics does not allow for definition of complex mechanisms like they have been proposed for fixed networks, the following four basic QoS classes are proposed:

a) The conversational class requires to preserve the time relation (variation) between information entities of a stream and puts stringent restrictions on the tolerable delay. The most well known example of this class is classical telephony, but with emerging use of Internet and multimedia a number of new applications, e.g. voice over IP or video conferencing tools, are expected to require this scheme.

b) The streaming class also demands for preservation of the timing relation between information entities of a stream but, as it tolerates higher end-toend delays and thus gives more flexibility to align the timing relation at the receiver, the acceptable delay variation is much higher than that of the conversational class. The streaming class applies to unidirectional transport of information with the most well known applications being audio and video distribution.

c) The interactive class addresses the QoS requirements of applications where an end-user interacts with remote equipment in order to acquire some data. The fundamental QoS characteristics of this class are to preserve the payload contend and to meet an end-to-end delay that is suitable for the request-response pattern of its' applications, like web browsing or automated database queries.

d) The background class is supposed to be used for applications where the destination is not expecting data to arrive within a certain time, like this is the case with delivery of emails or content of push services, e.g. news. The only QoS requirement of this class is to preserve the payload content.

In order to be able to accurately describe the QoS requirements of traffic belonging to one of the above classes, the 3GPP is further working on the specification of suitable QoS parameters, which are still subject to ongoing discussions:

1. The maximum bitrate defines the maximum number of bits delivered by UMTS and to UMTS at a service access point within a period of time divided by the duration of the period. Traffic is conformant with the maximum bitrate as long as it follows a token bucket algorithm where the token rate equals the maximum bitrate and bucket size equals maximum SDU size.

2. The guaranteed bitrate specifies the guaranteed number of bits delivered by UMTS within a period of time, divided by the duration of the period. The conformance definition provides a parameter to specify a bucket size greater than the maximum SDU size in order to deal with bursty traffic. 
However, for the 1999 release this parameter is fixed to 1 , so that the bucket size equals the maximum SDU size.

3. The boolean parameter delivery order indicates whether the UMTS bearer service shall provide in-sequence delivery or not.

4. The specification of the maximum SDU size is needed for admission control and policing.

5. The $S D U$ format information can be used to specify a list of possible SDU sizes, which allows for more efficient realization of the bearer service.

6. The $S D U$ error ratio indicates the fraction of SDUs lost or detected as erroneous. The purpose of this parameter is to configure the protocols, algorithms and error detection schemes, primarily in UTRAN.

7. The residual bit error ratio indicates the undetected bit error ratio in delivered SDUs and is used to configure the radio interface protocols, algorithms, and error detection coding.

8. The parameter delivery of erroneous $S D U$ s allows to specify if delivery of erroneous SDUs with or without an error indication, respectively, is wanted or not.

9. The transfer delay parameter indicates the maximum delay for $95^{\text {th }}$ percentile of the distribution of delay for all delivered SDUs.

10. The traffic handling priority allows to specify the relative importance of the SDUs belonging to one bearer compared to the SDUs of other bearers. As priority handling is a fundamental alternative to absolute guarantees for providing QoS, this parameter can not be used together with parameters specifying absolute guarantees.

11. The allocation / retention priority specifies the relative importance of one bearer to other bearers and is used for call admission control in case of scarce resources. In the 1999 release this parameter is planned as a subscription parameter, which will not be negotiated with the user terminal. These parameters are defined for both the UMTS and the radio access bearers and parameter value ranges are defined for each of them. Table 1 shows which parameter is relevant for each of the above QoS classes as well as the parameter ranges proposed so far for radio access bearers. Please note, that for radio access bearers there is one additional parameter source statistics descriptor, which permits to specify, that speech is the payload of a bearer, as this allows for calculation of a multiplexing gain for use in admission control of the radio access network. 
Table 1. Radio access bearer attributes and value ranges for each QoS class

\begin{tabular}{|c|c|c|c|c|}
\hline Traffic class & Conversational & Streaming & Interactive & Background \\
\hline $\begin{array}{l}\text { Maximum bitrate } \\
(\mathrm{kbit/s})\end{array}$ & $<2048$ & $<2048$ & $\begin{array}{l}<2048- \\
\text { overhead }\end{array}$ & $\begin{array}{l}<2048- \\
\text { overhead }\end{array}$ \\
\hline Delivery order & Yes / No & Yes / No & Yes / No & Yes / No \\
\hline $\begin{array}{l}\text { Maximum SDU size } \\
\text { (octets) }\end{array}$ & $<=1502$ & $<=1502$ & $<=1502$ & $<=1502$ \\
\hline $\begin{array}{l}\text { SDU format } \\
\text { information }\end{array}$ & to be done & to be done & & \\
\hline SDU error ratio & Yes / No / - & Yes / No/ - & Yes / No / - & Yes / No / - \\
\hline Residual bit error ratio & $\begin{array}{l}5^{*} 10^{-2}, 10^{-2}, 5^{*} 10^{-} \\
3,10^{-3}, 10^{-4}, 10^{-6}\end{array}$ & $\begin{array}{l}5^{*} 10^{-2}, 10^{-2}, \\
5^{*} 10^{-3}, 10^{-3}, \\
10^{-4}, 10^{-5}, 10^{-6}\end{array}$ & $\begin{array}{l}4^{*} 10^{-3}, 10^{-} \\
5,6^{*} 10^{-8}\end{array}$ & $\begin{array}{l}4^{*} 10^{-3}, 10^{-5} \\
6^{*} 10^{-8}\end{array}$ \\
\hline $\begin{array}{l}\text { Delivery of erroneous } \\
\text { SDUs }\end{array}$ & $\begin{array}{l}10^{-2}, 7^{*} 10^{-3}, 10^{-3} \\
10^{-4}, 10^{-5}\end{array}$ & $\begin{array}{l}10^{-1}, 10^{-2} \\
7^{*} 10^{-3}, 10^{-3} \\
10^{-4}, 10^{-5}\end{array}$ & $\begin{array}{l}10^{-3}, 10^{-4} \\
10^{-6}\end{array}$ & $10^{-3}, 10^{-4}, 10^{-6}$ \\
\hline Transfer delay (ms) & $80(\max )$. & 250 (max.) & & \\
\hline $\begin{array}{l}\text { Guaranteed bitrate } \\
(\mathrm{kbit} / \mathrm{s})\end{array}$ & $<2048$ & $<2048$ & & \\
\hline Traffic handling priority & & & $1,2,3$ & \\
\hline $\begin{array}{l}\text { Allocation / Retention } \\
\text { priority }\end{array}$ & $1,2,3$ & $1,2,3$ & $1,2,3$ & $1,2,3$ \\
\hline
\end{tabular}

\section{AAL-2 BASED TRANSPORT IN THE UTRAN}

During the last couple of years the ITU-T has been working on a set of recommendations $[13,14,15,16,17]$ defining an ATM adaptation layer that may be used for applications demanding a finer grained segmentation than that provided by existing adaptation layers, whose SDUs are at least 47 octets in size. The new adaptation layer AAL-2 allows for multiplexing of multiple communication streams into one or more ATM connections by multiplexing so called minicells, also referred to as AAL-2 CPS-Packets, containing a payload of 1 to 65535 octets into the cells of ATM connections. The 3GPP technical specification group for the radio access network (TSG RAN) has decided to use AAL-2 for transport between the radio network 
controllers (RNC) and Node Bs [1, 2, 3, 4] in order to allow for resource efficient transport of low-bitrate traffic in the radio access network.

AAL-2 is structured into multiple sublayers: the common part sublayer (CPS) and the service specific convergence sublayer (SSCS), which itself is structured into the service specific segmentation and reassembly (SSSAR), the service specific transmission error detection (SSTED), and the service specific assured data transfer (SSADT) sublayer. Only the CPS needs to be present in an AAL-2 entity, the use of the other sublayers depends on the applications' needs.

The AAL-2 minicell header consists of three octets and starts with an eight bit channel identifier (CID), allowing to multiplex up to 256 different AAL-2 connection into one ATM virtual circuit (VC). The following length indicator (LI) is one less than the number of octets in the CPS-Packet payload. Its' maximum value may be fixed to 44 or 63 , depending on the implementation (restricting the maximum to 44 may allow to re-use existing designs when building an AAL-2 switch). The field user-to-user indication (UUI) allows to signal 5 bits in the header of a minicell to the peer AAL-2 entity. It is followed by the field header error correction (HEC) which provides a 5 bit cyclic redundancy check code over the minicell header.

In order to insert minicells into the cells of an ATM-connection, AAL-2 entities assemble them into CPS-PDUs, each one filling exactly the payload of one ATM cell. The first field of a CPS-PDU (which is located directly after the ATM cell header) is the offset field (OSF) which carries the offset in octets to the first minicell header or the beginning of the pad field which is carried in this CPS-PDU where a value of zero indicates the position right after the parity bit $(P)$. In case, that there is neither a minicell header nor a pad field in this CPS-PDU it carries the value 47. The offset is used for minicell delineation. Between the offset field and the parity bit, a one bit serial number (SN) is located, which allows for detection of any uneven loss of CPS-PDUs. The payload of the CPS-PDU carries the minicells where minicells can arbitrarily cross the boundaries of CPS-PDUs within their length limits.

The ITU-T recommendation I.363.2 which standardizes AAL-2 doesn't deal in detail with questions related to QoS issues. However, it mentions to distinguish between different QoS-requirements of the AAL-2 user by the use of multiple service access points (SAPs, see also figure 2).

This situation gives rise to multiple questions concerning the realization of QoS in the context of the AAL-2 protocol:

- Which will be the QoS parameters or QoS classes offered to the service user of AAL-2 (i.e. definition of QoS at the AAL2 layer)? 


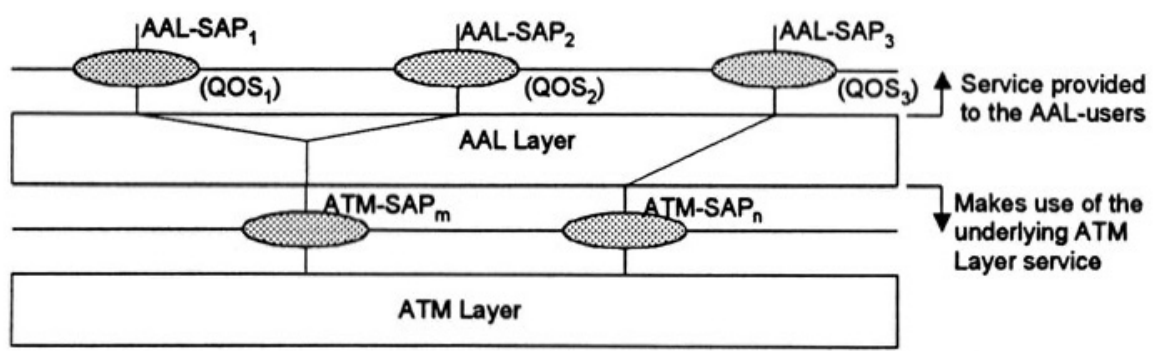

Figure 2: Relation between AAL-SAP and ATM-SAP (TTU-T Rec. I.363.2)

- What type of "guarantee" will be offered (statistic, as in the IP guaranteed service, but which is only provided as an indication associated with the connection, or deterministic, as in ATM on a per connection basis)

- Is there a need for a QoS negotiation or even re-negotiation at the AAL-2 layer, and if so, how might it be realized?

- How will this QoS specification be translated to the QoS offered by the ATM layer?

- What mechanisms can be used to ensure the QoS-guarantees given to the service user?

Basically, one has to distinguish between traffic parameters, that are descriptions of particular traffic aspects characterizing the traffic offered at a standardized interface, like peak cell rate, average cell rate, cell delay variation tolerances, burstiness, or peak duration, and quality of service (QoS) parameters, which characterize the transfer of traffic between two interfaces.

Concerning AAL-2 traffic parameters it seems reasonable to define them analogue to those defined for the ATM layer (cf. [19]): peak cell rate (PCR), cell delay variation tolerance (CDVT), sustainable cell rate (SCR), maximum burst size (MBS), etc. will have to be translated to peak minicell rate (PMR), minicell delay variation tolerance (MDVT), sustainable minicell rate (SMR), etc. Additionally, the AAL-2 traffic parameters should allow to specify the traffic streams properties concerning the size of exchanged minicells. Therefore, it seems obvious to add the mean and the maximum minicell size to the already existing traffic parameters.

In the same way as AAL-2 traffic parameters, the AAL-2 QoS parameters might best be defined analogous to the those of the ATM layer (see also [18] for detailed definitions), e.g. minicell error ratio (MER), minicell loss ration (MLR), minicell misinsertion rate (MMR), minicell transfer delay (MTD), and minicell delay variation (MDV).

It remains to be analyzed, how the guarantees given with an AAL-2 traffic contract can be assured by an AAL-2 entity. This is directly related to the means of traffic control deployed in the network, and particularly the realization of the scheduling, the buffer management as well as the bandwidth management in AAL-2. 


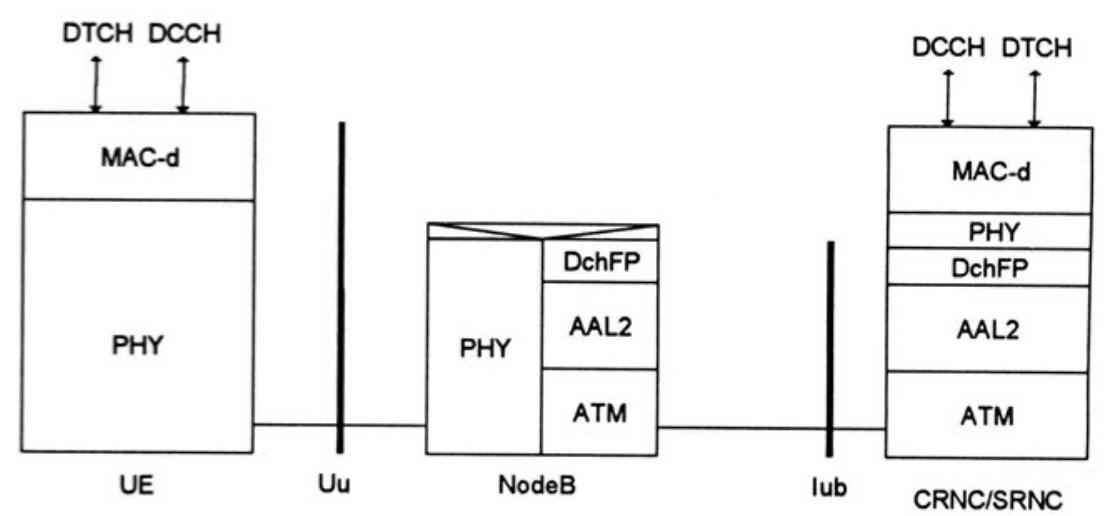

Figure 3: UTRAN protocol model for dedicated channels (3G TS 25.401)

However, when deployed in the UTRAN, this situation might be somehow simpler, as the protocol models of the UTRAN architecture reveal that the traffic to be transferred between two AAL-2 entities might be more homogenous, than the heterogeneous nature of the UMTS QoS classes seem to indicate at a first glance. Figure 3 depicts the protocol model for transport of dedicated channels between an RNC the Node Bs controlled by him.

As the RNC controls the radio resources of his Node Bs, the MAC layer processing, including the scheduling of heterogeneous communication streams, is handled by the RNC. This implies, that the purpose of a Node B and the corresponding AAL-2 connection to his RNC can be interpreted as "prolonging" the (upper part of the) physical layer between the mobile terminal and the RNC. In this respect, all traffic transported over AAL-2 in the UTRAN is in fact realtime traffic. This simplifies the constraints on AAL-2 in this scenario, as depending on the actual implementation it might not be necessary to provide sophisticated scheduling and buffer management mechanisms to cope with an arbitrary mix of realtime and non-realtime traffic.

The provision of quality of service in the transport network of the UTRAN has to serve two different purposes:

1. Satisfy the user: the requirements of the QoS classes defined for UMTS should met when transporting user data.

2. Make good resource utilization: this applies especially to the relatively rare and expensive radio resources and puts further constraints on the handling of traffic, as e.g. PDUs of the radio MAC need to reach the Node B on time, regardless of the QoS class of their content in order to avoid unused radio resources.

Summarizing, the answers to the questions, whether and how to distinguish between traffic with and without real time constraints on the level of AAL-2 will depend significantly on the design of the radio access network. 


\section{IP BASED TRANSPORT IN THE UTRAN}

A quite recent development in the standardization of $3^{\text {rd }}$ generation mobile communication systems is the investigation of an All-IP network architecture. This effort is conducted by the 3GPP2 consortium, which has been formed in reaction to the unwillingness of the ETSI to include other "non GSM" technologies in its' proposal for IMT-2000 [6].

The high level objectives of an All-IP network identified in a working report of 3GPP2 are [7]:

- to provide a unified (voice/data) wireless IP network that is interoperable with existing services, has gateways to legacy networks, and supports high capacity,

- to reuse existing radio network technology and to be independent of a specific air interface,

- to enable new services built on top of IP,

- to provide a global solution with an IP based infrastructure, as well as

- to maximize synergy and compatibility with existing standardization efforts.

Up to now, only vague ideas exist, how a $3^{\text {rd }}$ generation mobile communication system based on an All-IP architecture should look like. Nevertheless, it is interesting to point out some of the QoS and performance issues of an All-IP based versus an ATM/AAL-2 based solution.

Various investigations $[9,11,12,21,22,23]$ are showing the performance advantages of AAL- 2 for the transport of low bitrate voice data in comparison to prior ATM adaptation layers. Comparing AAL-2 to IP reveals firstly the differences in terms of header overhead. With AAL-2 every minicell comes with an overhead of 3 octets for up to 45/64 octets of payload plus additional 5 octets of overhead for every ATM cell, whereas IP can transport up to 65536 octets with only an overhead of 20 octets. So, IP will surely impose less overhead if the fraction of data traffic is high in comparison to voice traffic. However, overhead is just one performance aspect, as the ATM based approach might as well have difficulties to cope with very high data rates due to segmentation and reassembly processing.

Two architectures are currently considered. The first one is based on IP over MPLS with IETF differentiated services (DiffServ) as a framework of QoS. The second is based on native IP routing with DiffServ for the QoS.

\section{ALTERNATIVE ARCHITECTURES FOR USRAN}

UMTS involves a satellite segment which complements the terrestrial one to offer a global mobile communication service. The satellite component 
can play the same role of the access network which UTRAN presents in terrestrial UMTS segments and hence, will be referred to as UMTS Satellite Radio Access Network (USRAN). USRAN can be a backup option for terrestrial UTRAN in two cases. The first case arises in areas which are not covered by terrestrial networking infra-structures. The satellite component is then essential to relay the user equipment to the nearest UMTS or UMTScompatible network. The second case occurs when the terrestrial UMTS segment is saturated, and no resources are available for arriving or handed over calls. The satellite segment can then act as an umbrella cell to provide extra resources to the terrestrial segment.

The mobility management in the satellite context is handled differently from the terrestrial case. Indeed, the cell size in the satellite case, several hundred kilometres, is much larger than in the terrestrial case (few kilometres). Thus the handover (HO) operations in the satellite case occur mostly because of the satellite mobility (the LEO/MEO case).

Two possible architectural scenarios can be imagined to integrate the satellite component in the UMTS access network. The first scenario sees the satellite acting as a Node B in the USRAN, while in the second, the space segment, consisting of one or more satellites, supports the dual functionality of a Node B and an RNC. In both cases, the uplink radio channel connects the user equipment to the UMTS core network through the space segment, which can be a geo-stationary satellite or a constellation of Low or Medium Earth Orbit satellites.

\subsection{Satellite as Node B}

The satellite segment in this architecture offers the functions of a Node $B$ to the terrestrial UMTS segment. This scenario is most probable to encounter in areas with no terrestrial coverage and the satellite segment serves as a link between the user equipment and a remote RNC which is geographically out of reach of the user equipment. This implies that the satellite will act as a transceiver to support all the radio transmission functions, which are normally supported by the terrestrial Node B such as modulation, demodulation, rake receiver, coding etc. The radio transmission technology between the mobile and the satellite can be based on the ESA proposal [10] using Satellite Wideband CDMA SW-CDMA multiple access technique and operating in FDD mode with channel bandwidth of either 2.5 or $5 \mathrm{Mhz}$ in each direction. The proposed solution is fairly close to the WCDMA used with UTRAN. 


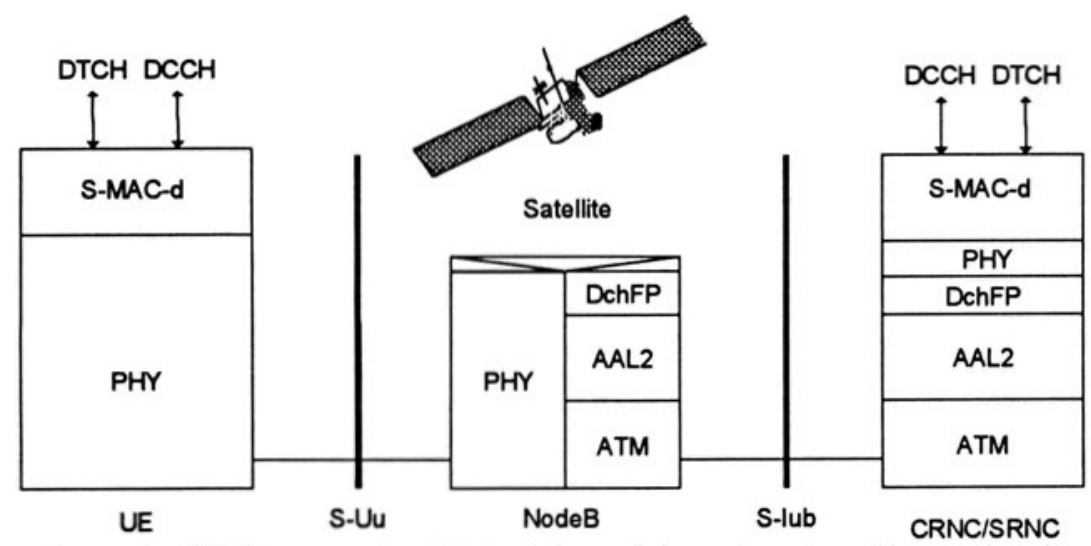

Figure 4: USRAN protocol model for dedicated channels with satellite as Node B

The particularity of the satellite link imposes additional precautions on the applied error detection and correction capabilities of the physical layer, between the terminal and the satellite in one hand and between the satellite and the RNC in the other hand, in order to keep the bit error rate comparable to the terrestrial segment and hence, preserve the quality of service parameters for the different services.

Figure 4 illustrates the protocol architecture when the satellite is playing the role of a Node B. The interface between user equipment and the satellite segment is referred to as $\mathrm{S}-\mathrm{Uu}$, while the interface between the satellite and the RNC is referred to as S-lub. The AAL2 used within the S-Iub protocol stack may include sublayers SSTED for the error detection and SSADT (with SSCOP) for error correction in order to ensure reliable minicell exchange mode. The QoS provided by the access network to the user requirements can be defined at the MAC layer as a set of Transfer Capabilities MTCs. Three MTCs can be defined: CBR, Bursty Data and Best Effort [20].

\subsection{Satellite as RNS}

In this architectural scenario, the satellite segment can support the double role of an umbrella cell for terrestrial UMTS segments, as well as a relay between user equipment and remote UMTS core networks. The functions of both, the Node B and the RNC will be implemented in the satellite, which will then act as a spatial radio access network. The satellite segment will then handle the mobility, as well as admission and call control functions, besides all the physical aspects of transmission on both up- and down-links. 


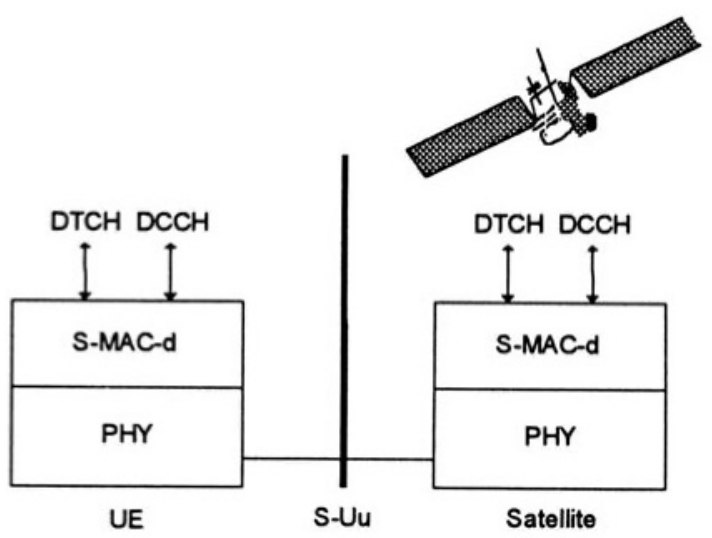

Figure 5: USRAN protocol model for dedicated channels with satellite as Node B

The protocol structure onboard the satellite is presented in figure 5. The satellite segment then ensures the functions of the physical, MAC, RLC, $\mathrm{RRC}$ and CC layers. The S-Uu interface relays the user equipment to the USRAN, while the S-Iu interface relays the space segment to the UMTS terrestrial core network. The satellite segment must be able to communicate directly with terrestrial RNCs, and hence the presence of the interface S-Iur. In addition, a satellite acting as USRAN must also be able to communicate directly with other satellites in the USRAN (in the presence of Inter-Satellite Links). Another interface intra-satellites must then be defined at this level. The MTCs are defined in the same manner than in the precedent architecture scenario.

\subsection{Comparison}

The first scenario is very similar with the UTRAN scheme. The complexity of the system in term of resource control is located within the terrestrial segment. The HO between terrestrial and spatial segments is simpler as this architecture corresponds to a hierarchical network. Because of the thorough control of satellite resources from the $\mathrm{RNC}$, the satellite needs practically to be owned by the UMTS operator, which may reduce its economical feasibility.

In the second scenario the complexity is completely located on the segment space. This would allow for a global satellite access operator who offers his services to multiple UMTS operators. In this case, however, the HO will correspond to a more complex roaming operation. 


\section{CONCLUSION}

The radio access network technology of the $3^{\text {rd }}$ generation mobile networks are anticipated to evolve towards a packet oriented architecture, as it is expected that the fraction of data traffic will increase in relation with mobile computing power increase. The ATM technology with AAL-2 might be seen as a first step in the $3^{\text {rd }}$ generation radio network evolution as it is build on the premise, that low bitrate voice transmission will be the dominant part of the traffic. IP technology including the ongoing work of the IETF on IP QoS is currently appearing as the next technological step to operate within fixed/mobile and terrestrial/satellite networks.

\section{REFERENCES}

[1] 3GPP Technical Specification Group Radio Acces Network (TSG RAN). UTRAN Overall Description. 3G TS 25.401.

[2] 3GPP TSG RAN. UTRAN Iu Interface, Data Transport and Transport Signalling. 3G TS 25.414.

[3] 3GPP TSG RAN. UTRAN Iur and Iub Interfaces, Data Transport and Transport Signalling for DCH Data Stream. 3G TS 25.426.

[4] 3GPP TSG RAN. UTRAN Iub Interface, General Aspects and Principles. 3G TS 25.430 .

[5] 3GPP TSG Services and System Aspects. Qos Concept and Architecture Release 1999). 3G TS 23.107.

[6] 3GPP2. Background on the $3^{\text {rd }}$ Generation Partnership Project 2. http://www.3gpp2.org/text/background.cfm, 1999.

[7] 3GPP2 TSG-S. All IP Adhoc Report to TSG-S - 01/00. S00ALLIP-20000106-024, January 2000.

[8] A. Adas. Traffic Models in Broadband Networks. Communications Magazine, pp. 8289, IEEE, July 1997.

[9] J. H. Baldwin, B. H. Bharucha, B. T. Doshi, S.. Dravida, S. Nanda. $A A L 2-A N e w$ ATM Adaptation Layer for Small Packet Encapsulation and Multiplexing. Bell Labs Technical Journal, Spring 1997.

[10] ESA. Wideband CDMA Option for the Satellite Component of IMT-2000 "SW-CDMA". ESA Proposal for a Candidate RTT. V. 1.0. June 1998.

[11] N. Gerlich, M. Ritter. Carrying CDMA Traffic over ATM using AAL-2: A Performance Study. Research Report No. 188, Department of Distributed Systems, University of Würzburg, November 1997.

[12] N. Gerlich, M. Menth. The Performance of AAL-2 Carrying CDMA Voice Traffic. Research Report No. 199, Department of Distributed Systems, University of Würzburg, April 1998.

[13] ITU-T. B-ISDN ATM Adaptation Layer specification: Type 2 AAL. Recommendation I.363.2, 1997.

[14] ITU-T. Segmentation and Reassembly Service Specific Convergence Sublayer for the AAL type 2. Recommendation I.366.1, 1998. 
[15] ITU-T. AAL Type 2 Service Specific Convergence Sublayer for Narrow-Band Services. Draft Recommendation I.366.2, March 2000.

[16] ITU-T. AAL Type 2 Service Specific Convergence Sublayer for Mobile. Draft Recommendation I.366.3, November 1999.

[17] ITU-T. AAL Type 2 Signalling Protocol (Capability Set 1). Recommendation Q.2630.1, 1999.

[18] ITU-T. B-ISDN ATM Layer Cell Transfer Performance. Recommendation I.356, October 1996.

[19] ITU-T. Traffic Control and Congestion Control in B-ISDN. Recommendation I.371, August 1996.

[20] H. Koraitim, S. Tohmé. Resource Allocation and Connection Admission Control in Satellite Networks. IEEE Journal on Selected Areas in Communications, February 1999.

[21] C. Liu, S. Munir, R. Jain, S. Dixit. Packing Density of Voice Trunking Using AAL 2. Globecom '99, Rio de Janeiro, Brazil, December 1999.

[22] K. Sriram, T. G. Lyons, Y. T. Wang. Anomalies Due to Delay and Loss in AAL-2 Packet Voice Systems: Performance Models and Methods of Mitigation. IEEE Journal on Selected Areas in Communications, January 1999.

[23] K. Sriram, Y. T. Wang. Voice over ATM using AAL-2 and Bit Dropping: Performance and Call Admission Control. IEEE Journal on Selected Areas in Communications, January 1999. 